\title{
Existence of $p$-adic quasi Gibbs measure for countable state Potts model on the Cayley tree
}

\author{
Farrukh Mukhamedov
}

Correspondence: far75m@yandex. ru

Department of Computational \& Theoretical Sciences, Faculty of Science, International Islamic University Malaysia, P.O. Box, 141, 25710, Kuantan, Pahang, Malaysia

\begin{abstract}
In the present article, we provide a new construction of measure, called $p$-adic quasi Gibbs measure, for countable state of $p$-adic Potts model on the Cayley tree. Such a construction depends on a parameter $\mathfrak{p}$ and wights. In particular case, i.e., if $\mathfrak{p}=\exp _{p}$, the defined measure coincides with $p$-adic Gibbs measure. In this article, under some condition on weights we establish the existence of $p$-adic quasi Gibbs measures associated with the model. Note that this condition does not depend on values of the prime $p$. An analogues fact is not valid when the number of spins is finite.

Mathematics Subject Classification: 46S10; 82B26; $12 \mathrm{~J} 12$.
\end{abstract}

Keywords: countable, $p$-adic numbers, Potts model, Gibbs measure, uniqueness

\section{Introduction}

Interest in the physics of non-Archimedean quantum models [1-5] is based on the idea that the structure of space-time for very short distances might conveniently be described in terms of non-Archimedean numbers. One of the ways to describe this violation of the Archimedean axiom, is the using $p$-adic analysis. Numerous applications of this analysis to mathematical physics have been proposed in [4,6-8]. It is known [8] that a number of $p$-adic models in physics cannot be described using ordinary Kolmogorov's probability theory. New probability models- $p$-adic probability models were investigated in [9-11]. In [12-14], the theory of stochastic processes with values in $p$-adic and more general non-Archimedean fields having probability distributions with non-Archimedean values has been developed. This gives a possibility to develop the theory of statistical mechanics in the context of the $p$-adic theory, since it lies on the base of the theory of probability and stochastic processes. The central problems of that theory [15] is the study of infinite-volume Gibbs measures corresponding to a given Hamiltonian, which also includes a description of the set of Gibbs measures. In most cases such analysis depend on a specific properties of Hamiltonian, and complete description is often a difficult problem. This problem, in particular, relates to a phase transitions problem.

In $[16,17]$, a notion of ultrametric Markovianity, which describes independence of contributions to random field from different ultrametric balls has been introduced, and showed that Gaussian random fields on general ultrametric spaces (which were related with hierarchical trees), which were defined as a solution of pseudodifferential stochastic

(c) 2012 Mukhamedov; licensee Springer. This is an Open Access article distributed under the terms of the Creative Commons Attribution License (http://creativecommons.org/licenses/by/2.0), which permits unrestricted use, distribution, and reproduction in any medium, provided the original work is properly cited. 
equation, satisfy the Markovianity. Some applications of the results to replica matrices, related to general ultrametric spaces have been investigated in [18] (see also [19]).

In $[20,21]$, we have developed of $p$-adic probability theory approaches to study $q+1$ state nearest-neighbor $p$-adic Potts model on Cayley tree (see [22]). We constructed infinite volume $p$-adic Gibbs measures for the mentioned model, and moreover, established the existence of a phase transition (here the phase transition means the existence of two distinct $p$-adic Gibbs measures for the given model). Further, in [23] we have introduced a new kind of $p$-adic measures for the mentioned model, called $p$-adic quasi Gibbs measure. For such a model, we investigated a phase transition phenomena from the associated dynamical system point of view. Namely, we established that if $q$ is not divisible by $p$, then there occurs the quasi phase transition. Note that such kind of measures present more natural concrete examples of $p$-adic Markov processes (see [12], for definitions). In [24-26], we investigated a countable state $p$-adic Potts model on the Cayley tree, ${ }^{a}$ and provided a construction of $p$-adic Gibbs measures which depends on weights $\lambda$. Moreover, the uniqueness of such measures under certain conditions to the weight was proved.

In this article, we continue our investigations on countable state $p$-adic Potts model. Namely, we are going to provide a new construction of measure, called $p$-adic quasi Gibbs measure, for the mentioned model on the Cayley tree. Such a construction depends on a parameter $\mathfrak{p}$ and wights $\{\lambda(i)\}_{i \in \mathbb{N}}$. In particular case, i.e., if $\mathfrak{p}=\exp _{p}$, the defined measure coincides with $p$-adic Gibbs measure (see $[24,25]$ ). In this article, under some condition on weights we establish the existence of $p$-adic quasi Gibbs measures associated with the model. Note that this condition does not depend on values of the prime $p$. An analogues fact is not valid when the number of spins is finite. Moreover, our result extends the previous proved ones in $[25,26]$. Note that in comparison to a real case, in a $p$-adic setting, à priori the existence of such kind of measures for the model is not known, since there is not much information on topological properties of the set of all $p$-adic measures defined even on compact spaces. However, in the real case, there is the so called the Dobrushin's Theorem [27,28] which gives a sufficient condition for the existence of the Gibbs measure for a large class of Hamiltonians. Note that when states are finite, then the corresponding $p$-adic Potts models on the same trees have been studied in [23].

\section{Preliminaries}

Fix a prime number $p$, which throughout the article will be a fixed $>3$, and let $\mathbb{Q}_{p}$ denotes the field of $p$-adic filed, formed by completing $\mathbb{Q}$ with respect to the unique absolute value satisfying $|p|=1 / p$. The absolute value $|\cdot|$, is non- Archimedean, meaning that it satisfies the ultrametric triangle inequality $|x+y|_{p} \leq \max \left\{|x|_{p},|y|_{p}\right\}$.

Given $a \in \mathbb{Q}_{p}$ and $r>0$ put

$$
B(a, r)=\left\{x \in \mathbb{Q}_{p}:|x-a|_{p}<r\right\}, \quad S(a, r)=\left\{x \in \mathbb{Q}_{p}:|x-a|_{p}=r\right\} .
$$

The $p$-adic exponential is defined by

$$
\exp _{p}(x)=\sum_{n=0}^{\infty} \frac{x^{n}}{n !}
$$

which converges for $x \in B\left(0, p^{-1 /(p-1)}\right)$. 
Lemma 2.1. [24]If $\left|a_{i}\right|_{p} \leq 1,\left|b_{i}\right|_{p} \leq 1, i=1, \ldots, n$, then

$$
\left|\prod_{i=1}^{n} a_{i}-\prod_{i=1}^{n} b_{i}\right|_{p} \leq \max _{i \leq i \leq n}\left\{\left|a_{i}-b_{i}\right|_{p}\right\}
$$

Note the basics of $p$-adic analysis, $p$-adic mathematical physics are explained in $[5,29]$.

Let $(X, \mathscr{B})$ be a measurable space, where $\mathscr{B}$ is an algebra of subsets $X$. A function $\mu$ : $\mathscr{B} \rightarrow \mathbb{Q}_{p}$ is said to be a $p$-adic measure if for any $A_{1}, \ldots, A_{n} \subset \mathscr{B}$ such that $A_{i} \cap A_{j}=\varnothing$ $(i \neq j)$ the equality holds

$$
\mu\left(\bigcup_{j=1}^{n} A_{j}\right)=\sum_{j=1}^{n} \mu\left(A_{j}\right) .
$$

A $p$-adic measure is called a probability measure if $\mu(X)=1$. For more detail information about $p$-adic measures we refer to $[9,10,30]$.

Let $\Gamma^{k}=(V, L)$ be a semi-infinite Cayley tree of order $k \geq 1$ with the root $x^{0}$ (whose each vertex has exactly $k+1$ edges, except for the root $x^{0}$, which has $k$ edges). Here $V$ is the set of vertices and $L$ is the set of edges. The vertices $x$ and $y$ are called nearest neighbors and they are denoted by $l=\langle x, y\rangle$ if there exists an edge connecting them. A collection of the pairs $\left\langle x, x_{1}>, \ldots,<x_{d-1}, y>\right.$ is called a path from the point $x$ to the point $y$. The distance $d(x, y), x, y \in V$, on the Cayley tree, is the length of the shortest path from $x$ to $y$. Let us set

$$
W_{n}=\left\{x \in V \mid d\left(x, x^{0}\right)=n\right\}, V_{n}=\bigcup_{m=1}^{n} W_{m}, L_{n}=\left\{l=<x, y>\in L \mid x, y \in V_{n}\right\} .
$$

The set of direct successors of $x$ is defined by

$$
S(x)=\left\{y \in W_{n+1}: d(x, y)=1\right\}, x \in W_{n} .
$$

Observe that any vertex $x \neq x^{0}$ has $k$ direct successors and $x^{0}$ has $k+1$.

Now we are going to introduce a coordinate structure in $\Gamma^{k}$. Every vertex $x$ (except for $\left.x_{0}\right)$ of $\Gamma^{k}$ has coordinates $\left(i_{1}, \ldots, i_{n}\right)$, here $i_{m} \in\{1, \ldots, k\}, 1 \leq m \leq n$ and for the vertex $x_{0}$ we put. $\varnothing$. Namely, the symbol $\varnothing$ constitutes level 0 and the sites $\left(i_{1}, \ldots, i_{n}\right)$ form level $n$ of the lattice. In this notation for $x \in \Gamma^{k}, x=\left(i_{1}, \ldots, i_{n}\right)$ we have

$$
S(x)=\{(x, i): 1 \leq i \leq k\},
$$

here $(x, i)$ means that $\left(i_{1}, \ldots, i_{w}, i\right)$.

Let us define on $\Gamma^{k}$ a binary operation o: $\Gamma^{k} \times \Gamma^{k} \rightarrow \Gamma^{k}$ as follows: for any two elements $x=\left(i_{1}, \ldots, i_{n}\right)$ and $y=\left(j_{1}, \ldots, j_{m}\right)$ put

$$
x \circ y=\left(i_{1}, \ldots, i_{n}\right) \circ\left(j_{1}, \ldots, j_{m}\right)=\left(i_{1}, \ldots, i_{n}, j_{1}, \ldots, j_{m}\right)
$$

and

$$
x \circ x^{0}=x^{0} \circ x=\left(i_{1}, \ldots, i_{n}\right) \circ(0)=\left(i_{1}, \ldots, i_{n}\right) .
$$

By means of the defined operation $\Gamma^{k}$ becomes a noncommutative semigroup with a unit. 
Using this semigroup structure one defines translations $\tau_{g}: \Gamma^{k} \rightarrow \Gamma^{k}, g \in \Gamma^{k}$ by

$$
\tau_{g}(x)=g \circ x .
$$

It is clear that $\tau_{(0)}=i d$.

Similarly, by means of $\tau_{g}$ one can define translations $\tilde{\tau}_{g}: L \rightarrow L$ of $L$. Namely,

$$
\tilde{\tau}_{g}(<x, y>)=<\tau_{g}(x), \tau_{g}(y)>.
$$

Let $G \subset \Gamma^{k}$ be a sub-semigroup of $\Gamma^{k}$ and $h: \Gamma^{k} \rightarrow \mathbb{R}$ be a function defined on $\Gamma^{k}$. We say that $h$ is G-periodic if $h\left(\tau_{g}(x)\right)=h(x)$ for all $g \in G$ and $x \in \Gamma^{2}$. Any $\Gamma^{k}$-periodic function is called translation invariant. Put

$$
G_{k}=\left\{x \in \Gamma^{k}: d\left(x, x^{0}\right) \equiv 0(\bmod k)\right\}, k \geq 2
$$

One can check that $G_{k}$ is a sub-semigroup with a unit.

\section{The $\boldsymbol{p}$-adic Potts model}

We consider the $p$-adic Potts model where spin takes values in the set $\Phi=\{0,1,2, \ldots$, and is assigned to the vertices of the tree $\Gamma^{k}=(V, L)$. A configuration $\sigma$ on $V$ is then defined as a function $x \in V \rightarrow \sigma(x) \in \Phi$; in a similar manner one defines configurations $\sigma_{n}$ and $\sigma^{(n)}$ on $V_{n}$ and $W_{n}$, respectively. The set of all configurations on $V$ (resp. $V_{n}, W_{n}$ ) coincides with $\Omega=\Phi^{V}$ (resp. $\Omega_{V_{n}}=\Phi^{V_{n}}, \Omega_{w_{n}}=\Phi^{W_{n}}$ ). One can see that $\Omega_{V_{n}}=\Omega_{V_{n-1}} \times \Omega_{W_{n}}$. Using this, for given configurations $\sigma_{n-1} \in \Omega_{V_{n-1}}$ and $\sigma^{(n)} \in \Omega_{W_{n}}$ we define their concatenations by

$$
\sigma_{n-1} \vee \sigma^{(n)}=\left\{\left\{\sigma_{n}(x), x \in V_{n-1}\right\},\left\{\sigma^{(n)}(y), y \in W_{n}\right\}\right\} .
$$

It is clear that $\sigma_{n-1} \vee \sigma^{(n)} \in \Omega_{V_{n}}$.

The Hamiltonian $H_{n}: \Omega_{V_{n}} \rightarrow \mathbb{Q}_{p}$ of the $p$-adic countable state Potts model has the form

$$
H_{n}(\sigma)=\sum_{<x, y>\in L_{n}} N_{x y} \delta_{\sigma(x), \sigma(\gamma)}, \quad n \in \mathbb{N},
$$

here $\sigma \in \Omega_{w}, N_{x y} \in \mathbb{N}(x, y \in V)$ and $\delta$ is the Kronecker symbol.

We are going to construct $p$-adic quasi Gibbs measures for the model.

Let us consider a function $\mathbf{h}: x \in V \rightarrow \mathbf{h}_{x}=\left\{h_{i, x}\right\}_{i \in \Phi} \in \mathbb{Q}_{p}^{\Phi}$ of $x \in V \backslash\left\{x^{(0)}\right\}$. Here $\mathbb{Q}_{p}^{\Phi}=\left\{\left\{x_{i}\right\}_{i \in \Phi}: x_{i} \in \mathbb{Q}_{p}\right\}$.

Fix a sequence $\lambda \in \mathbb{Q}_{p}^{\Phi}$ such that

$$
|\lambda(n)|_{p} \rightarrow 0 \text { as } n \rightarrow \infty .
$$

Such kind of sequences are called weights.

Given $n=1,2, \ldots$ define a $p$-adic probability measure $\mu_{\mathrm{h}}^{(n)}$ on $\Omega_{V_{n}}$ by

$$
\mu_{\mathbf{h}}^{(n)}\left(\sigma_{n}\right)=\frac{1}{Z_{n}^{(\mathbf{h})}} \mathfrak{p}^{H_{n}\left(\sigma_{n}\right)} \prod_{x \in W_{n}} h_{\sigma_{n}(x), x} \prod_{x \in V_{n}} \lambda\left(\sigma_{n}(x)\right)
$$


where $\mathfrak{p}$ is any $p$-adic number such that $|\mathfrak{p}|_{p} \leq 1$. In particular, it could be $\mathfrak{p}=p$. Here, as before, $\sigma_{n}: x \in V_{n} \rightarrow \sigma_{n}(x) \in \Phi$ and $Z_{n}^{(\mathrm{h})}$ is the corresponding partition function:

$$
Z_{n}^{(\mathbf{h})}=\sum_{\sigma \in \Omega_{V_{n}}} \mathfrak{p}^{H_{n}(\sigma)} \prod_{x \in W_{n}} h_{\sigma(x), x} \prod_{x \in V_{n}} \lambda(\sigma(x)) .
$$

One of the central results of the theory of probability concerns a construction of an infinite volume distribution with given finite-dimensional distributions, which is called Kolmogorov's Theorem [31]. Therefore, in this article we are interested in the same question but in a $p$-adic context. More exactly, we want to define a $p$-adic probability measure $\mu$ on $\Omega$ which is compatible with defined ones $\mu_{\mathrm{h}}^{(n)}$, i.e.,

$$
\mu\left(\sigma \in \Omega: \sigma \mid V_{n}=\sigma_{n}\right)=\mu_{\mathrm{h}}^{(n)}\left(\sigma_{n}\right), \text { for all } \sigma_{n} \in \Omega_{V_{n}}, n \in \mathbb{N} .
$$

In general, à priori the existence such a kind of measure $\mu$ is not known, since there is not much information on topological properties, such as compactness, of the set of all $p$-adic measures defined even on compact spaces. ${ }^{\mathrm{b}}$ Note that certain properties of the set of $p$-adic measures has been studied in [32,33], but those properties are not enough to prove the existence of the limiting measure. Therefore, at a moment, we can only use the $p$-adic Kolmogorov extension Theorem (see [12,34]) which based on so called compatibility condition for the measures $\mu_{\mathrm{h}}^{(n)}, n \geq 1$, i.e.,

$$
\sum_{\omega \in \Omega_{W_{n}}} \mu_{\mathbf{h}}^{(n)}\left(\sigma_{n-1} \vee \omega\right)=\mu_{\mathbf{h}}^{(n-1)}\left(\sigma_{n-1}\right)
$$

for any $\sigma_{n-1} \in \Omega_{V_{n-1}}$. This condition according to the theorem implies the existence of a unique $p$-adic measure $\mu$ defined on $\Omega$ with a required condition (3.5). Note that more general theory of $p$-adic measures has been developed in [35].

So, if for some function $\mathbf{h}$ the measures $\mu_{\mathbf{h}}^{(n)}$ satisfy the compatibility condition, then there is a unique $p$-adic probability measure, which we denote by $\mu_{\mathbf{h}}$, since it depends on $\mathbf{h}$. Such a measure $\mu_{\mathbf{h}}$ is said to be a p-adic quasi Gibbs measure corresponding to the $p$-adic Potts model. By $Q \mathcal{G}(H)$ we denote the set of all $p$-adic quasi Gibbs measures associated with functions $\mathbf{h}=\left\{\mathbf{h}_{x} \in V\right\}$. If there are at least two distinct $p$-adic quasi Gibbs measures $\mu, v \in Q \mathcal{G}(H)$ such that $\mu$ is bounded and $v$ is unbounded, then we say that a phase transition occurs. By another words, one can find two different functions $\mathbf{s}$ and $\mathbf{h}$ defined on $\mathbb{N}$ such that there exist the corresponding measures $\mu_{\mathbf{s}}$ and $\mu_{\mathbf{h}}$, for which one is bounded, another one is unbounded. If there are two different functions $\mathbf{s}$ and $\mathbf{h}$ defined on $\mathbb{N}$ such that there exist the corresponding measures $\mu_{\mathbf{s}}$, $\mu_{\mathbf{h}}$, and they are bounded, then we say there is a quasi phase transition.

Remark 3.1. Note that in [24] we considered the following sequence of $p$-adic measures defined by

$$
\mu_{\mathbf{h}}^{(n)}\left(\sigma_{n}\right)=\frac{1}{\tilde{Z}_{n}^{(\mathbf{h})}} \exp _{p}\left\{H_{n}\left(\sigma_{n}\right)+\sum_{x \in W_{n}} h_{\sigma_{n}(x), x}\right\} \prod_{x \in V_{n}} \lambda\left(\sigma_{n}(x)\right)
$$


here as usual $\tilde{Z}_{n}^{(h)}$ is the corresponding normalizing factor. A limiting $p$-adic measures generated by (3.7) was called p-adic Gibbs measure. Such kind of measures have been studied in [24].

Now let us find for what kind of functions $\mathbf{h}=\left\{\mathbf{h}_{x}: x \in V\right\}$ the measures defined by (3.3) would satisfy the compatibility condition (3.6). The following statement describes conditions on $\mathbf{h}$ guaranteeing the compatibility condition for the measures $\mu_{\mathbf{h}}^{(n)}$.

Theorem 3.1. The measures $\mu_{\mathrm{h}}^{(n)}, n=1,2, \ldots$ satisfy the compatibility condition (3.6) if and only if for any $x \in V \backslash\left\{x^{(0)}\right\}$ the following equation holds:

$$
\hat{h}_{i, x}=\frac{\lambda(i)}{\lambda(0)} \prod_{y \in S(x)} F_{i}\left(\hat{h}_{y} ; \theta_{x y}\right), \quad i \in \mathbb{N}
$$

here and below $\theta_{x y}=\mathfrak{p}^{N_{x y}}$, a vector $\hat{\mathbf{h}}=\left\{\hat{h}_{i}\right\}_{i \in \mathbb{N}} \in \mathbb{Q}_{p}^{\mathbb{N}}$ is defined by a vector $\mathbf{h}=\left\{h_{i}\right\}$ $i \in \Phi$ as follows

$$
\hat{h}_{i}=\frac{h_{i} \lambda(i)}{h_{0} \lambda(0)}, \quad i \in \mathbb{N}
$$

and a mapping $F: \mathbb{Q}_{p}^{\mathbb{N}} \rightarrow \mathbb{Q}_{p}^{\mathbb{N}}$ is $F(\mathbf{x} ; \theta)=\left\{F_{i}(\mathbf{x} ; \theta)\right\}_{i \in \mathbb{N}}$ with

$$
F_{i}(\mathbf{x} ; \theta)=\frac{(\theta-1) x_{i}+\sum_{j=1}^{\infty} x_{j}+1}{\sum_{j=1}^{\infty} x_{j}+\theta}, \mathbf{x}=\left\{x_{i}\right\}_{i \in \mathbb{N}}, i \in \mathbb{N} .
$$

The proof consists of checking the condition (3.6) for the measures (3.3) (cp. $[24,36])$.

Remark 3.2. Note that thanks to non-Archimedeanity of the norm $|\cdot|_{p}$ the series $\sum_{k=1}^{\infty} x_{k}$ converges iff the sequence $\left\{x_{n}\right\}$ converges to 0 (see [29]). Therefore, in what follows we should assume that $\sum_{k=1}^{\infty} \hat{h}_{k, x}$ converges, otherwise the Equation (3.8) has no sense.

Remark 3.3. In what follows, without loss of generality, we may assume that $h_{0}=1$ and $\lambda(0)=1$. Otherwise, in (3.3) we multiply and divide the expression on the right hand side by $\prod_{x \in W_{n}} h_{0, x} \prod_{x \in V_{n}} \lambda(0)$, and after replacing $h_{i}$ by $h_{i} / h_{0}$ and $\lambda(k)$ by $\lambda(k) / \lambda$ (0), respectively, we get the desired equality.

Observation 3.1. Here we are going to underline a connection between $q$-state Potts model with the defined one. First recall that $q$-state Potts model is defined by the same Hamiltonian (3.1), but with the state space $\Phi_{q}=\{0,1, \ldots, q-1\}$. Similarly, one can define $p$-adic quasi Gibbs measures for the $q$-state Potts model, here instead of the weight $\{\lambda(i)\}$ we will take a collection $\{\lambda(0), \lambda(1), \ldots, \lambda(q-1)\} \subset \mathbb{Q}_{p}$.

Now consider countable Potts model with a weight $\{\lambda(i)\}$ such that

$$
\lambda(k)=0 \text { for all } k \geq q, q>1 .
$$

In this case the corresponding $p$-adic quasi Gibbs measures will coincide with those of $q$-state Potts model. Indeed, let 


$$
\begin{aligned}
& \Omega^{c}=\left\{\sigma \in \Omega: \exists j \in \mathbb{Z}_{+}: \sigma(j) \geq q\right\} \\
& \Omega^{(q)}=\left\{\sigma \in \Omega: \sigma(j) \leq q-1 \forall j \in \mathbb{Z}_{+}\right\}
\end{aligned}
$$

It is clear that $\Omega^{(q)}=\Phi_{q}^{\mathbb{Z}_{+}}$. Let $\mu$ be a $p$-adic quasi Gibbs measure of the countable Potts model with the given weight corresponding to a solution $\mathbf{h}_{n}=\left\{h_{i, n}\right\}_{i \in \Phi}$ of (3.8). From the definition (3.3) we see that the restriction of $\mu$ to $\Omega^{c}$ is zero, i.e., $\mu\left\lceil\Omega^{c}=0\right.$. Moreover, from (3.8) and (3.11) we conclude that $h_{i, n}=0$ for all $i \geq q$. This means that vectors $\mathbf{h}_{n}^{(q)}=\left\{h_{i, n}\right\}_{i \in \Phi_{q}}$ will be a solution of (3.8) corresponding to the $q$-state Potts model. Therefore, the restriction of $\mu$ to $\Omega^{(q)}$ coincides with $p$-adic quasi Gibbs measure of $q$-state Potts model with a weight $\{\lambda(0), \lambda(1), \ldots, \lambda(q-1)\}$ corresponding to a solution of $\mathbf{h}_{n}^{(q)}$.

Hence, we conclude that under the condition (3.11) all $p$-adic quasi Gibbs measures corresponding to countable Potts model are described by those measures of $q$-state Potts model.

\section{Existence of $\boldsymbol{p}$-adic quasi Gibbs measure}

In this section, we are going to provide a condition for the existence of the $p$-adic quasi Gibbs measure.

Taking into account Remark 3.2, we consider the following space

$$
c_{0}=\left\{\left\{x_{n}\right\}_{n \in \mathbb{N}} \subset \mathbb{Q}_{p}:\left|x_{n}\right|_{p} \rightarrow 0 \text { as } n \rightarrow \infty\right\}
$$

with a norm $\|x\|=\max _{n}\left|x_{n}\right|_{p}$. Define

$$
\mathbf{B}_{r}=\left\{\left\{x_{n}\right\}_{n \in \mathbb{N}} \in c_{0}:\|x\| \leq r\right\}, r>0 .
$$

It is clear that $\mathbf{B}_{r}$ is a closed set.

Lemma 4.1. Let $|\theta|_{p} \geq 1$. Then

$$
\left|F_{i}(\mathbf{x}, \theta)-F_{i}(\mathbf{y}, \theta)\right|_{p} \leq\|\mathbf{x}-\mathbf{y}\| \text { for every } \mathbf{x}, \mathbf{y} \in \mathbf{B}_{\delta} .
$$

Proof. Let $\mathbf{x}=\left(x_{i}\right), \mathbf{y}=\left(y_{i}\right) \in \mathbf{B}_{\delta}$, then it is clear that $\left|x_{i}\right|_{p} \leq \delta$ and $\left|y_{i}\right|_{p} \leq \delta$ for all $i \in$ $\mathbb{N}$.

Therefore, the strong triangle inequality implies that

$$
\left|\sum_{j=1}^{\infty} x_{j}+\theta\right|_{p}=|\theta|_{p}\left|\sum_{j=1}^{\infty} y_{j}+\theta\right|_{p}=|\theta|_{p} .
$$

From (3.10) one gets

$$
\begin{aligned}
\left|F_{i}(\mathbf{x}, \theta)-F_{i}(\mathbf{y}, \theta)\right|_{p} & =\frac{|\theta-1|_{p}}{|\theta|_{p}^{2}}\left|\left(\sum_{j=1}^{\infty} x_{j}+\theta\right)\left(x_{i}-y_{i}\right)+\left(1-x_{i}\right) \sum_{j=1}^{\infty}\left(x_{j}-y_{j}\right)\right|_{p} \\
& \leq \frac{1}{|\theta|_{p}} \max \left\{|\theta|_{p}\left|x_{i}-y_{i}\right|_{p^{\prime}}\left|1-x_{i}\right|_{p}\left|\sum_{j=1}^{\infty}\left(x_{j}-y_{j}\right)\right|_{p}\right\} \\
& \leq \max _{i}\left|x_{i}-y_{i}\right|_{p} \\
& =\|\mathbf{x}-\mathbf{y}\| .
\end{aligned}
$$


This completes the proof. $\square$

Before going to Equation (3.8), let us first enumerate $S(x)$ for any $x \in V$ as follows $S$ $(x)=\left\{x_{1}, \ldots, x_{k}\right\}$, here as before $S(x)$ is the set of direct successors of $x$ (see (2.1)). Using this enumeration one can rewrite (3.8) by

$$
\hat{h}_{i, x}=\lambda(i) \prod_{m=1}^{k} F_{i}\left(\hat{\mathbf{h}}_{x m}, \theta_{i}\right), \quad i \in \mathbb{N} \text {, for every } x \in V \backslash\left\{x^{(0)}\right\} .
$$

Now we can formulate the main result.

Theorem 4.2. Assume that $N_{x y} \leq 0$ for all $\langle x, y\rangle \in$ L, i.e., $\left|\theta_{x y}\right|_{p} \geq 1$ and for $\lambda$ the condition

$$
|\lambda(i)|_{p} \leq \delta \quad \forall_{i} \in \mathbb{N},
$$

is satisfied. Then any two solution of (4.3) belonging to $\mathbf{B}_{\delta}$ coincides with each other.

Proof. Assume that $\hat{\mathbf{h}}=\left\{\mathbf{h}_{x}, x \in V\left\{x^{0}\right\}\right\}, \hat{\mathbf{s}}=\left\{\hat{\mathbf{s}}_{x}, x \in V\left\{x^{0}\right\}\right\}$ be two solutions of (4.3). Now we are going to show that they coincide with each other. Indeed, let $x \in V \backslash\left\{x^{(0)}\right\}$ be an arbitrary vertex. Using Lemma 2.1 from (4.3) one can find

$$
\left.\left|\hat{h}_{i, x}-\hat{s}_{i, x}\right|_{p} \leq|\lambda(i)|_{p} \max _{1 \leq m \leq k}\left\{\mid F_{i}\left(\hat{\mathbf{h}}_{x m}\right)\right)_{i}-\left.F_{i}\left(\hat{\mathbf{s}}_{x_{m}}\right)\right|_{p}\right\},
$$

which with (4.1) and (4.4) implies that

$$
\left\|\hat{\mathbf{h}}_{x}-\hat{\mathbf{s}}_{x}\right\| \leq \delta \max _{1 \leq m \leq k}\left\{\left\|\hat{\mathbf{h}}_{x_{m}}-\hat{\mathbf{s}}_{x_{m}}\right\|\right\}
$$

Now take an arbitrary $\varepsilon>0$. Let $n_{0} \in \mathbb{N}$ such that $1 / p^{n_{0}}<\varepsilon$. Iterating (4.5) $n_{0}$ times one gets $\| \hat{\mathbf{h}}_{x}-\hat{\mathbf{s}}_{x}||<\varepsilon$.

Hence, from the arbitrariness of $\varepsilon$ we obtain $\hat{\mathbf{h}}_{x}=\hat{\mathbf{s}}_{x}$ for every $x \in V \backslash\left\{x^{(0)}\right\}$. This completes the proof. $\square$

The provided theorem states, if Equation (4.3) has solution belonging to $\mathbf{B}_{\delta}$, then it is unique. But, in general, we do not whether the equation has a solution or not. Below, we provide a sufficient condition for the existence of solution of (4.3).

Remark 4.1. The proved Theorem extends the main results of $[25,26]$ to more general kind of measures, since there it was taken $\mathfrak{p}=\exp _{p}$.

\subsection{Homogeneous case}

In this subsection, we shall assume that $N_{x y}:=N$ for all $\langle x, y\rangle \in €$. Namely, we want to consider homogeneous case.

Recall that a function $\mathbf{h}=\left\{\mathbf{h}_{x}\right\}_{x \in V \backslash\left\{x^{0}\right\}}$ is called translation-invariant if $\mathbf{h}_{\tau_{x}(y)}=\mathbf{h}_{y}$ for all $x, y \in V \backslash\left\{x^{0}\right\}$. Let us restrict ourselves to the description of translation-invariant solutions of (3.8), namely $\mathbf{h}_{x}=\mathbf{h}\left(=\left(h_{0}, h_{1}, \ldots,\right)\right)$ for all $x \in V$.

Define the following mapping

$$
\left(\mathcal{F}_{\theta}(\mathbf{x})\right)_{i}=\lambda(i)\left(F_{i}(\mathbf{x}, \theta)\right)^{k}, \quad i \in \mathbb{N},
$$

where $\mathbf{x}=\left\{x_{n}\right\} \in c_{0}$. Here, $\theta=\mathfrak{p}^{N}$. Due to Remark 3.2, the mapping $\mathscr{F}_{\theta}$ is well defined. 
Theorem 4.3. Let $N \leq 0$, i.e., $|\theta|_{p} \geq 1$. Assume that for $\lambda$ the condition (4.4) is satisfied. Then $\mathscr{F}_{\theta}\left(\mathbf{B}_{\delta}\right) \subset \mathbf{B}_{\delta}$, and

$$
\left\|\mathcal{F}_{\theta}(\mathbf{x})-\mathcal{F}_{\theta}(\mathbf{y})\right\| \leq \delta\|\mathbf{x}-\mathbf{y}\| \text { for every } \mathbf{x}, \mathbf{y} \in \mathbf{B}_{\delta} .
$$

Proof. Let $\mathbf{x}=\left(x_{i}\right), \mathbf{y}=\left(y_{i}\right) \in \mathbf{B}_{\delta}$. Then from (3.10) with (4.2) we have

$$
\left|F_{i}(\mathbf{x}, \theta)\right|_{p}=\frac{\left|\theta x_{i}+\sum_{j=1, j \neq i}^{\infty} x_{j}+1\right|_{p}}{|\theta|} \leq \frac{\max \left\{|\theta|_{p}\left|x_{i}\right|_{p}, 1\right\}}{|\theta|_{p}} \leq 1
$$

this with (4.6) yields that $\widetilde{\mathscr{F}}_{\theta}\left(\mathbf{B}_{\delta}\right) \subset \mathbf{B}_{\delta}$.

Now using (4.1), (4.6), and (4.8) we obtain

$$
\begin{aligned}
\left\|\mathcal{F}_{\theta}(\mathbf{x})-\mathcal{F}_{\theta}(\mathbf{y})\right\| & =\max _{i}|\lambda(i)|_{p} \max _{i}\left|\left(F_{i}(\mathbf{x}, \theta)\right)^{k}-\left(F_{i}(\mathbf{y}, \theta)\right)^{k}\right|_{p} \\
& \leq \delta\left|F_{i}(\mathbf{x}, \theta)-F_{i}(\mathbf{y}, \theta)\right|_{p} \max _{i}\left|\sum_{\ell=0}^{k-1} F_{i}^{k-\ell}(\mathbf{x}, \theta) F_{i}^{\ell}(\mathbf{y}, \theta)\right|_{p} \\
& \leq \delta\|\mathbf{x}-\mathbf{y}\| .
\end{aligned}
$$

This completes the proof. $\square$

Remark 4.2. We should stress that if $N>0$ then the similar methods are no longer applicable for $F_{\theta}$, therefore, it needs other kind of techniques. So, such a case will be considered elsewhere.

Now thanks to Lemma 4.3 we can apply the fixed point theorem to $\mathscr{H}_{\theta}$, which implies the existence of unique fixed point $\mathbf{x}_{0} \in \mathbf{B}_{\delta}$. This, according to Theorem 4.2, means that there exists a unique solution of (3.8). Hence, due to Theorem 3.1, such a solution defines the $p$-adic quasi Gibbs measure $\mu_{0}$.

Remark 4.3. Let us emphasize the following notes:

(a) Note that in [23] we have proved for the $q+1$-state Potts model the $p$-adic quasi Gibbs measure is unique if $q$ and $p$ are relatively prime. Therefore, the proved Theorem 4.2 shows the difference between finite and countable state Potts models. Moreover, in the real case such kind of result is unknown (see [36,37]).

(b) We also should stress that the condition (4.4) is important. If we replace $\delta$ with 1 , then Theorem 4.2 may not be valid. Namely, in that case it may occur a quasi phase transition. Indeed, if $\lambda(1)=\lambda(2)=1$ and $\lambda(k)=0$ for every $k \geq 3$, then clearly (4.4) is not satisfied. On the other hand, our model reduces to 3 -state Potts model. For such a model in [23] the existence of the quasi phase transition has been proved at $p=2$.

\subsection{Periodic case}

In this section, we consider when $N_{x y}$ is $G_{m}$-periodic. This means that $N_{\tau_{g}(x)} \tau_{\tau_{g}(y)}=N_{x y}$ for all $g \in G_{m}$ and $x, y \in \Gamma^{m}$. Therefore, let us denote

$$
\theta_{i}=\mathfrak{p}^{N_{x y}}, \quad \text { if } d\left(x, x^{0}\right) \equiv i(\bmod m), i=0, \ldots, m-1 .
$$


We want to find $G_{m}$-periodic solution of (3.8). Recall that a function $\mathbf{h}=\left\{\mathbf{h}_{x}\right\}_{x \in V \infty \backslash\left\{x^{0}\right\}}$ is called $G_{m}$-periodic if $\mathbf{h}_{\tau g(x)}=\mathbf{h}_{x}$ for all $g \in G_{m}, x \in V \backslash\left\{x^{0}\right\}$. Note that if $\mathbf{h}=\left\{\mathbf{h}_{x}\right\}$ is a $G_{k}$-periodic, then it can be defined by a $k$-collection of vectors $\left(\mathbf{h}_{0}, \ldots, \mathbf{h}_{k-1}\right)$, where $\mathbf{h}_{i}=\left\{h_{j}^{(i)}\right\}_{i \in \mathbb{N}}$, i.e., $\mathbf{h}_{x}=\mathbf{h}_{i}$, if $d\left(x, x^{0}\right) \equiv i(\bmod m), i=0, \ldots, k-1$.

Then Equation (3.8) is reduced to the following one

$$
\left\{\begin{array}{l}
h_{j}^{(i)}=\lambda_{j}\left(F_{j}\left(\mathbf{h}^{(i+1)}, \theta_{i+1}\right)\right)^{k}, i=0, \ldots, m-2 \\
h_{j}^{(m-1)}=\lambda_{j}\left(F_{j}\left(\mathbf{h}^{(0)}, \theta_{0}\right)\right)^{k},
\end{array}\right.
$$

Hence, define the following mapping

$$
(\mathcal{H}(\mathbf{x}))=\mathcal{F}_{\theta_{m-1}}\left(\mathcal{F}_{\theta_{m-2}}\left(\cdots\left(\mathcal{F}_{\theta_{0}}(\mathbf{x})\right) \cdots\right)\right)
$$

where $\mathbf{x}=\left\{x_{n}\right\} \in c_{0}$.

It is clear that the fixed point of (4.10) defines a solution of (4.9)

Theorem 4.4. Let $\left|\theta_{i}\right|_{p} \geq 1, i=0, \ldots, m-1$. Assume that for $\lambda$ the condition (4.4) is satisfied. Then $\mathscr{H}\left(\mathbf{B}_{\delta}\right) \subset \mathbf{B}_{\delta}$, and

$$
\|\mathcal{H}(\mathbf{x})-\mathcal{H}(\mathbf{y})\| \leq \delta^{m}\|\mathbf{x}-\mathbf{y}\| \text { for every } \mathbf{x}, \mathbf{y} \in \mathbf{B}_{\delta} .
$$

The proof immediately follows from Theorem 4.3.

Consequently, Theorem 4.4 allows us to apply the fixed point theorem to $\mathscr{H}$, which implies the existence of unique fixed point $\mathbf{x}_{0} \in \mathbf{B}_{\delta}$. This, according to Theorem 4.2, means that there exists a unique solution of (3.8). Hence, due to Theorem 3.1, such a solution defines the $p$-adic quasi Gibbs measure $\mu_{m}$.

\section{Conclusion}

In the present study, we have provided a new construction of measure, called $p$-adic quasi Gibbs measure, for countable state $p$-adic Potts model on the Cayley tree. Note that the construction depends on a parameter $\mathfrak{p}$ and wights $\{\lambda(i)\}_{i \in \mathrm{N}}$. In particular case, i.e., if $\mathfrak{p}=\exp _{p}$, the defined measure coincides with $p$-adic Gibbs measure (see $[24,25])$. In this article, under some condition on weights we proved the existence of $p$-adic quasi Gibbs measures associated with the model. Note that this condition does not depend on values of the prime $p$. Moreover, our result extends the previous proved ones in $[25,26]$. An analogues fact is not valid when the number of spins is finite. We should stress that when states are finite and $\rho=p$, then the corresponding $p$-adic quasi Gibbs measures have been investigated in [23].

\section{Endnotes}

${ }^{\mathrm{a}}$ The classical (real value) counterparts of such models were considered in [15,36]. ${ }^{\mathrm{b}} \mathrm{In}$ the real case, when the state space is compact, then the existence follows from the compactness of the set of all probability measures (i.e., Prohorov's Theorem). When the state space is non-compact, then there is a Dobrushin's Theorem [27,28] which gives a sufficient condition for the existence of the Gibbs measure for a large class of Hamiltonians. 


\section{Acknowledgements}

The present study had been done within the IIUM grant EDW B11-159-0637. The author also acknowledged the Junior Associate scheme of the Abdus Salam International Centre for Theoretical Physics, Trieste, Italy.

\section{Competing interests}

The author declares that they have no competing interests.

Received: 8 February 2012 Accepted: 8 May 2012 Published: 8 May 2012

\section{References}

1. Areféva, IYa, Dragovic, B, Frampton, PH, Volovich, IV: The wave function of the Universe and p-adic gravity. Int J Modern Phys A. 6(24):4341-4358 (1991). doi:10.1142/S0217751X91002094

2. Beltrametti, E, Cassinelli, G: Quantum mechanics and p-adic numbers. Found Phys. 2, 1-7 (1972). doi:10.1007/ BF00708614

3. Freund, PGO, Witten, E: Adelic string amplitudes. Phys Lett B. 199, 191-195 (1987). doi:10.1016/0370-2693(87)91357-8

4. Marinary, E, Parisi, G: On the $p$-adic five point function. Phys Lett B. 203, $52-56$ (1988). doi:10.1016/0370-2693(88)915699

5. Vladimirov, VS, Volovich, IV, Zelenov, El: $p$-adic Analysis and Mathematical Physics. World Scientific, Singapour (1994)

6. Avetisov, VA, Bikulov, AH, Kozyrev, SV: Application of $p$-adic analysis to models of spontaneous breaking of the replica symmetry. J Phys A: Math Gen. 32, 8785-8791 (1999). doi:10.1088/0305-4470/32/50/301

7. Khrennikov, AYu: $p$-adic Valued Distributions in Mathematical Physics. Kluwer Academic Publisher, Dordrecht (1994)

8. Khrennikov, AYu: Non-Archimedean Analysis: Quantum Paradoxes, Dynamical Systems and Biological Models. Kluwer Academic Publisher, Dordrecht (1997)

9. Khrennikov, AYu: $p$-adic valued probability measures. Indag Math NS. 7, $311-330$ (1996). doi:10.1016/0019-3577(96) 83723-2

10. Khrennikov, AYu, Nilsson, M: p-adic Deterministic and Random Dynamical Systems. Kluwer, Dordreht (2004)

11. Khrennikov, AYu, Yamada, S, van Rooij, A: Measure-theoretical approach to $p$-adic probability theory. Annals Math Blaise Pascal. 6, 21-32 (1999)

12. Khrennikov, A, Ludkovsky, S: Stochastic processes on non-Archimedean spaces with values in non-Archimedean fields. Markov Process Relat Fields. 9, 131-162 (2003)

13. Ludkovsky, SV: Non-Archimedean valued quasi-invariant descending at infinity measures. Int J Math Math Sci. 2005(23):3799-3817 (2005). doi:10.1155/JMMS.2005.3799

14. Khrennikov, AYu: Limit behaviour of sums of independent random variables with respect to the uniform $p$-adic distribution. Statis \& Probab Lett. 51, 269-276 (2001). doi:10.1016/50167-7152(00)00158-9

15. Georgii, HO: Gibbs Measures and Phase Transitions. Walter de Gruyter, Berlin (1988)

16. Khrennikov, AYu, Kozyrev, SV: Wavelets on ultrametric spaces. Appl Comput Harmonic Anal. 19, 61-76 (2005). doi:10.1016/j.acha.2005.02.001

17. Khrennikov, AYu, Kozyrev, SV: Ultrametric random field. Infin Dimens Anal Quantum Probab Relat Top. 9, 199-213 (2006). doi:10.1142/S0219025706002317

18. Khrennikov, AYu, Kozyrev, SV: Replica symmetry breaking related to a general ultrametric space I, II, III. Physica A 359, 222-240 (2006). 359, 241-266 (2006); 378, 283-298 (2007)

19. Del Muto, M, Figà-Talamanca, A: Diffusion on locally compact ultrametric spaces. Expo Math. 22, 197-211 (2004). doi:10.1016/50723-0869(04)80005-7

20. Mukhamedov, FM, Rozikov, UA: On Gibbs measures of $p$-adic Potts model on the Cayley tree. Indag Math N S. 15, 85-100 (2004). doi:10.1016/50019-3577(04)90007-9

21. Mukhamedov, FM, Rozikov, UA: On inhomogeneous $p$-adic Potts model on a Cayley tree. Infin Dimens Anal Quantum Probab Relat Top. 8, 277-290 (2005). doi:10.1142/S0219025705001974

22. Wu, FY: The Potts model. Rev Mod Phys. 54, 235-268 (1982). doi:10.1103/RevModPhys.54.235

23. Mukhamedov, FM: On $p$-adic quasi Gibbs measures for $q+1$-state Potts model on Cayley tree. $p$-adic Numbers Ultametric Anal Appl. 2, 241-251 (2010). doi:10.1134/S2070046610030064

24. Khrennikov, A, Mukhamedov, F, Mendes, JFF: On p-adic Gibbs measures of countable state Potts model on the Cayley tree. Nonlinearity. 20, 2923-2937 (2007). doi:10.1088/0951-7715/20/12/010

25. Khrennikov, A, Mukhamedov, F: On uniqueness of Gibbs measure for p-adic countable state Potts model on the Cayley tree. Nonlinear Anal Theor Methods Appl. 71, 5327-5331 (2009). doi:10.1016/..na.2009.04.021

26. Mukhamedov, F: On existence of generalized Gibbs measures for one dimensional $p$-adic countable state Potts model. Proc Steklov Inst Math. 265, 165-176 (2009). doi:10.1134/S0081543809020163

27. Dobrushin, RL: The problem of uniqueness of a Gibbsian random field and the problem of phase transitions. Funct Anal Appl. 2(2):302-312 (1968)

28. Dobrushin, RL: Prescribing a system of random variables by conditional distributions. Theor Probab Appl. 15, 458-486 (1970). doi:10.1137/1115049

29. Koblitz, N: $p$-adic numbers, $p$-adic analysis and zeta-function. Springer, Berlin (1977)

30. van Rooii, A: Non-archimedean Functional Analysis. Marcel Dekker, New York (1978)

31. Shiryaev, AN: Probability. Nauka, Moscow (1980)

32. Katsaras, AK: On spaces of $p$-adic vector measures. p-Adic Numbers Ultrametric Anal Appl. 1, 190-203 (2009). doi:10.1134/S2070046609030029

33. Katsaras, AK: On p-adic vector measures. J Math Anal Appl. 365, 342-357 (2010). doi:10.1016/j.jmaa.2009.10.059

34. Ganikhodjaev, NN, Mukhamedov, FM, Rozikov, UA: Phase transitions of the Ising model on $\boldsymbol{\nabla}$ in the $p$-adic number field. Uzbek Mat J , 4: 23-29 (1998). Russian

35. Katsaras, AK: Extensions of p-adic vector measures. Indag Math NS. 19, 579-600 (2008). doi:10.1016/S0019-3577(08) 80022-5 
36. Ganikhodjaev, NN, Rozikov, UA: The Potts model with countable set of spin values on a Cayley tree. Lett Math Phys. 75, 99-109 (2006). doi:10.1007/s11005-005-0032-8

37. Ganikhodjaev, NN: The Potts model on $\mathbb{\nabla}^{d}$ with countable set of spin values. J Math Phys. 45, 1121-1127 (2004). doi:10.1063/1.1643194

doi:10.1186/1029-242X-2012-104

Cite this article as: Mukhamedov: Existence of $p$-adic quasi Gibbs measure for countable state Potts model on the Cayley tree. Journal of Inequalities and Applications 2012 2012:104.

Submit your manuscript to a SpringerOpen ${ }^{\odot}$ journal and benefit from:

- Convenient online submission

$\checkmark$ Rigorous peer review

- Immediate publication on acceptance

- Open access: articles freely available online

- High visibility within the field

- Retaining the copyright to your article

Submit your next manuscript at $\boldsymbol{s p r i n g e r o p e n . c o m ~}$ 